\title{
Best Practices in Rubella outbreak response in an International School in Nakuru County, Kenya
}

\author{
Kiptoo $\mathrm{E}^{1 \star}$, Itumbi $\mathbf{M}^{2}$, Ngari $\mathbf{R}^{3}$ and King'ori $\mathbf{S}^{1}$ \\ ${ }^{\prime}$ Department of Health Services Nakuru, Kenya \\ ${ }^{2}$ Molo Sub County Hospital, Kenya \\ ${ }^{3}$ Molo Sub County Health Office, Kenya
}

\section{Article Info}

\section{*Corresponding author:}

Elizabeth Kiptoo

Department of Health Services

Nakuru, Kenya

Tel: 254722337018

E-mail: izkiptoo@gmail.com

Received: June 19, 2017

Accepted: June 30, 2017

Published: July 7, 2017

Citation: Kiptoo E, Itumbi M, Ngari R, King'ori S. Best Practices in Rubella outbreak response in an International School in Nakuru County, Kenya. Madridge J Vaccines. 2017; 1(1): 19-23. doi: $10.18689 / \mathrm{mjv}-1000105$

Copyright: ( 2017 The Author(s). This work is licensed under a Creative Commons Attribution 4.0 International License, which permits unrestricted use, distribution, and reproduction in any medium, provided the original work is properly cited.

Published by Madridge Publishers

\begin{abstract}
The World Health Organization estimates that 1.5 million deaths among children under 5 years are due to vaccine preventable diseases, representing 17 per cent of underfive child mortality worldwide. Until recently Kenya did not have a rubella immunization program or a surveillance system for congenital Rubella Syndrome. Rubella Cases are being detected using measles case based surveillance system. World Health Organization recommended that countries including Kenya should conduct a wide age range campaign with MR vaccine, 6 months prior to its absorption into routine. The Kenya Ministry of Health with World Health Organization and UNICEF conducted measles Rubella catch up immunization campaign in May 2016 targeting children 9 months to 14 years. Nakuru County vaccinated $102 \%$ while Molo sub county where the rubella outbreak occurred vaccinated 95\%. Turi International School which has an eligible population of 1200 children 9 months to 14 years was totally missed out during the vaccination exercise due to vaccine hesitancy by the school management. Four months later rubella outbreak occurred affecting a total of 20 children aged between 2 years and 12 years. The Objective of this study is to demonstrate the best practices in rubella outbreak response and to document the need to prioritize efforts to address Challenges of vaccine refusal in future vaccination campaigns. The study was done in Molo Sub County in Nakuru County. Case definition was used to identify suspected cases. Data was collected in an excel line list and analyzed using SPSS version 20. Chi square was used to measure the association between the risk factor and the occurrence of rubella. Samples were analysed in KEMRI lab. Majority of the cases were from St Andrews school Turi, Children aged between 5-14 years were mostly affected. Those who did not receive the vaccine during the campaign were mostly affected. There is strong association between the occurrence of rubella (Chi 28.863, p 0.025 ) and non vaccination during the May 2016 MR Vaccination campaign. Following the outbreak, The county Rapid response team was convened, Surveillance intensified, health workers skills updated, Isolation ward was established, social mobilization and advocacy meetings were conducted. Finally, 5 days vaccination was conducted targeting children 9 months to 14 years. A total of $14,137(116 \%)$ children out of the 12,130 were vaccinated and the outbreak was controlled within two weeks.
\end{abstract}

Keywords: Rubella; Outbreak; Best practice; Vaccine refusal.

Abbreviations: CHMT: County Health Management Team; CRS: Congenital Rubella Syndrome; EPI: Expanded Program on Immunization; KEMRI: Kenya Medical Research Institute; MR: Measles Rubella; SPSS: Statistical Package for Social Science; UNICEF: United Nations Children Fund; VHP: Vaccine Hesitant Parents; VPDs: Vaccine Preventable Diseases; WHO: World Health Organization. 


\section{Introduction}

Rubella is an acute, contagious viral infection. In children, the disease is usually mild, with symptoms including a rash, low fever $\left(<39^{\circ} \mathrm{C}\right)$, nausea and mild conjunctivitis [1]. The rash, which occurs in 50-80\% of cases, usually starts on the face and neck before progressing down the body, and lasts 1-3 days. Swollen lymph glands behind the ears and in the neck are the most characteristic clinical feature [2].

Rubella has serious consequences in pregnant women causing fetal death or congenital defects known as congenital rubella syndrome (CRS) [3].When a woman is infected with the rubella virus early in pregnancy, she has a $90 \%$ chance of passing the virus on to her fetus. This can cause miscarriage, stillbirth or severe birth defects known as CRS. Infants with CRS may excrete the virus for a year or more [4]. Children with CRS can suffer hearing impairments, eye and heart defects and other lifelong disabilities, including autism, diabetes mellitus and thyroid dysfunction - many of which require costly therapy, surgeries and other expensive care [5]. The highest risk of CRS is in countries where women of childbearing age do not have immunity to the disease (either through vaccination or from having had rubella).

The World Health Organization (WHO) estimates that 1.5 million deaths among children under 5 years are due to vaccine preventable diseases (VPDs), representing 17 per cent of under-five child mortality worldwide [6]. Measles, mumps, and rubella are viral infections that are Preventable through vaccination programs. Under a national Expanded Program on Immunization (EPI) [7]. Until recently Kenya did not have a rubella immunization program or a surveillance system for congenital Rubella Syndrome (CRS). Rubella Cases are being detected using measles case based surveillance system [8].

World Health Organization recommended that countries including Kenya should conduct a wide age range campaign with MR vaccine, 6 months prior to its absorption into routine [10]. The Kenya Ministry of Health with World Health Organization (WHO) and UNICEF conducted measles Rubella (MR) catch up immunization campaign in May 2016 targeting children 9 months to 14 years in Kenya as part of Global Measles and rubella (MR) control strategies this is in line with the global Measles rubella elimination strategic plan 2012 to 2030 [9]. The ministry of Health $(\mathrm{MOH})$ vaccinated $98 \%$ of the targeted group countrywide. Nakuru County vaccinated $102 \%$ and Molo Sub County where the rubella outbreak occurred vaccinated 95\% [11]. Turi International School which has an eligible population of 1200 children 9 months to 14 years was totally missed out during the vaccination exercise due to vaccine refusal by the school management. Four months later rubella outbreak occurred in the school and the neighbourhood affecting a total of 20 children aged between 2 years and 12 years. By the time this outbreak occurred Kenya had not introduced this vaccine into routine expanded program on immunization (EPI).

The purpose of this study is to demonstrate the best practices in early case detection, investigation and prompt intervention that led to interruption of the circulation of rubella virus within a short period and to document the need to prioritize efforts to address Challenges of vaccine refusal from the start in future vaccination campaigns.

\section{Methodology}

\section{Study Area}

The study was carried out in Molo Sub County in Nakuru County which is located 50 kilometers away west of Nakuru town. Molo is one of the 11 sub counties in Nakuru County. It is administratively divided into 5 wards and covers an area of 478.79 square kilometers. It has a population of 146,977 people.

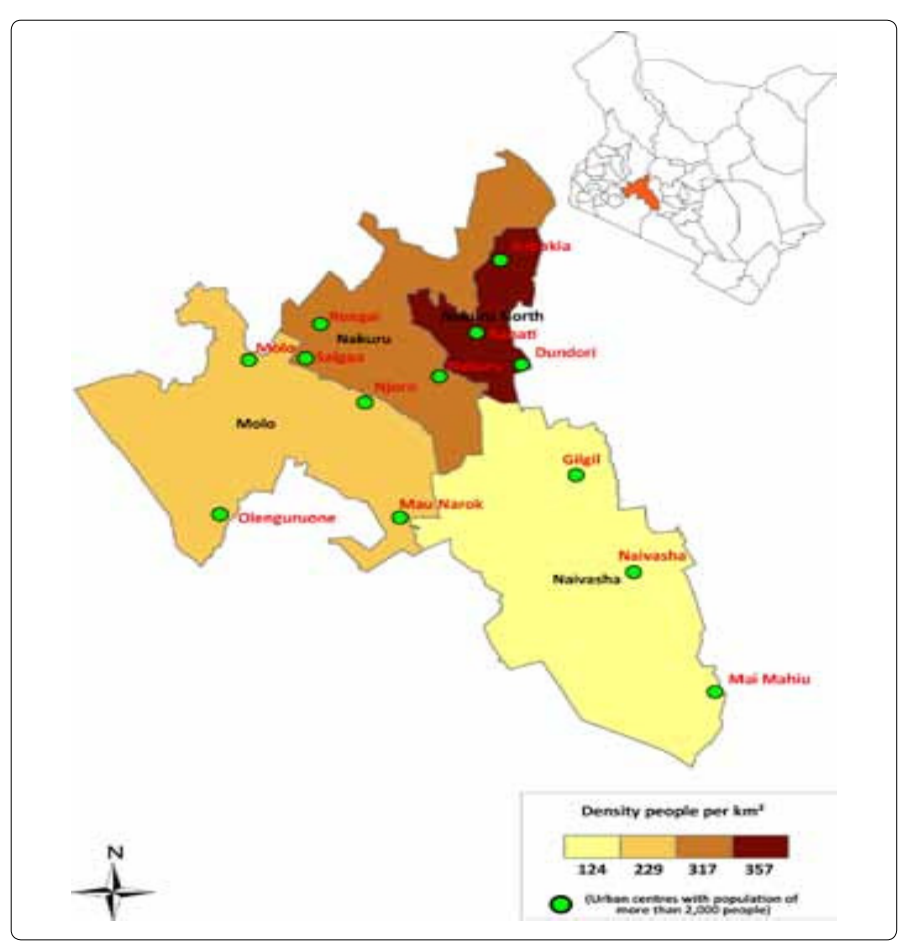

Figure 1. Map of Nakuru County

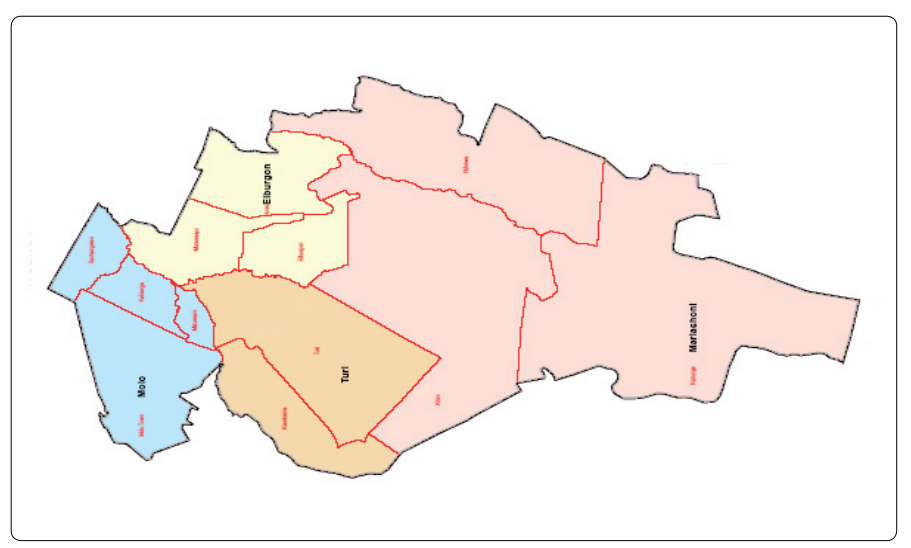

Figure 2. Map of Molo Sub County

\section{Study design}

This is retrospective descriptive records review of cases of rubella that were reported and investigated during rubella outbreak in the month of October 2016.

\section{Laboratory investigation}

Blood samples were collected from the first 5 suspected 
cases for serological confirmation. As per integrated disease surveillance guidelines in Kenya, For Surveillance for Non measles febrile rash illness, all specimens are first tested for measles- specific immunoglobulin (igM) antibody using standard enzyme linked immunosorbent essay. Because all the samples tested negative for measles igM, the same sera were later tested for presence of rubella specific igM antibody using standard enzyme linked immunosorbent essay. The laboratory testing was performed at Kenya Medical Research Institute (KEMRI) laboratory which is an accredited laboratory by world Health Organization.

Routinely once at least 3 or more suspected cases are confirmed as positive for either Measles or rubella igM in the laboratory then it is no longer necessary to test other samples. The subsequent cases are line listed as rubella cases as long as they are epidemiologically linked to the confirmed cases.

\section{Case Definition}

A suspected case is a person with maculopapular rash, fever and any one of the following; Cough coryza or conjunctivitis from the affected institution and the surrounding areas. A confirmed case was one that tested positive for rubella igM.

\section{Data Collection}

Data was entered into an outbreak line listing form. The line list captured ages, sexes, date of onset and date seen at the health facility, vaccination status, no of vaccine doses, residence, specimen collected and outcome.

\section{Data Analysis}

Data Analysis was done using SPSS version 20 Chi square tests for categorical variables was used to establish association of relevant risk factors with the occurrence of Rubella. All confidence intervals $(\mathrm{Cl})$ were maintained at $95 \%$. Statistical significance was defined at $p \leq 0.05$. $P$ value of $\leq 0.05$ was considered significant.

\section{Ethical Approval}

This was part of routine integrated disease surveillance, investigation and response therefore there was no need for ethical clearance.

\section{Results and Discussion}

This outbreak is the second largest outbreak occurring in Nakuru County and the first to occur four months after the MR vaccination campaign. All the 5 samples taken for laboratory confirmation tested positive for Rubella igM. Fifteen other cases were epidemiologically linked to the confirmed cases and therefore line listed. The index case was a 12 year old Ugandan boy who left Uganda on $28^{\text {th }}$ august 2016, had onset on $10^{\text {th }}$ September 201(Fig.3)6, 12 days after leaving his home country Uganda. This shows that he was exposed while in Uganda; most likely Uganda is the source of rubella infection according to the epidemiological information.

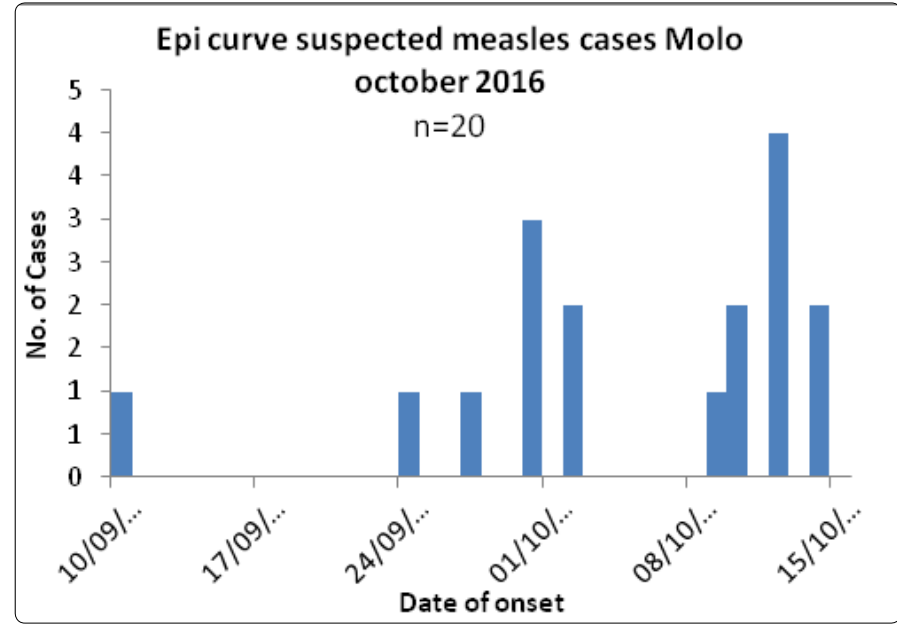

Figure 3. Epi curve of Rubella cases

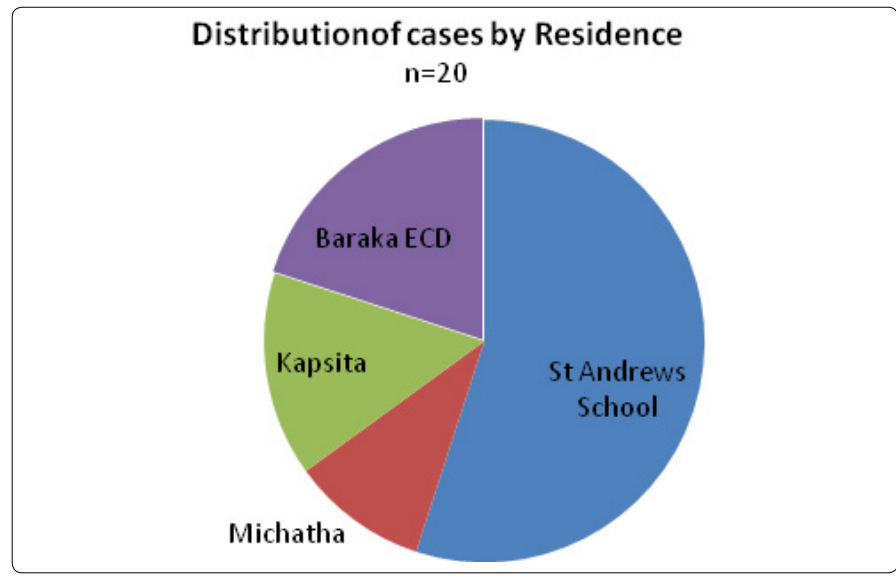

Figure 4. Rubella cases by residnce

Majority [11] of the cases were from St Andrews school Turi. All the cases from Turi were not vaccinated. Others were from its neighbourhoods.

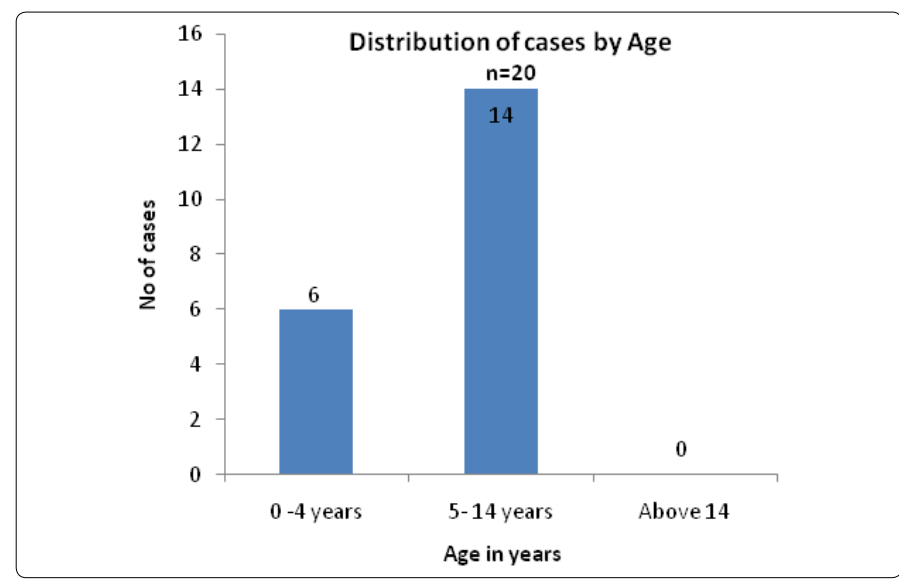

Figure 5. Distribution of rubella cases by Age

Children 2 years to 14 years were affected with the majority being in the age bracket of 5 to 14 years. All the cases were within the MR vaccination age group. 


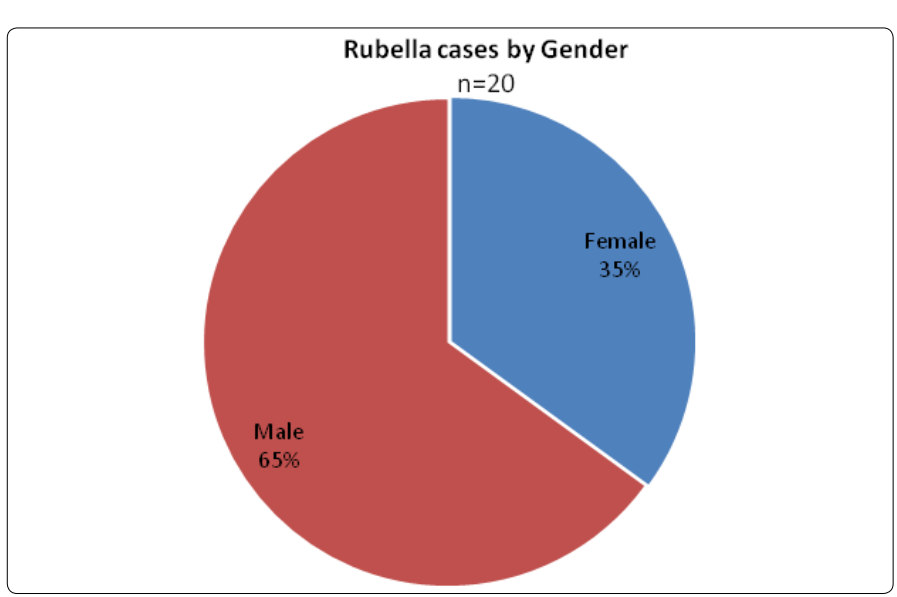

Figure 6. Rubella Cases by Gender

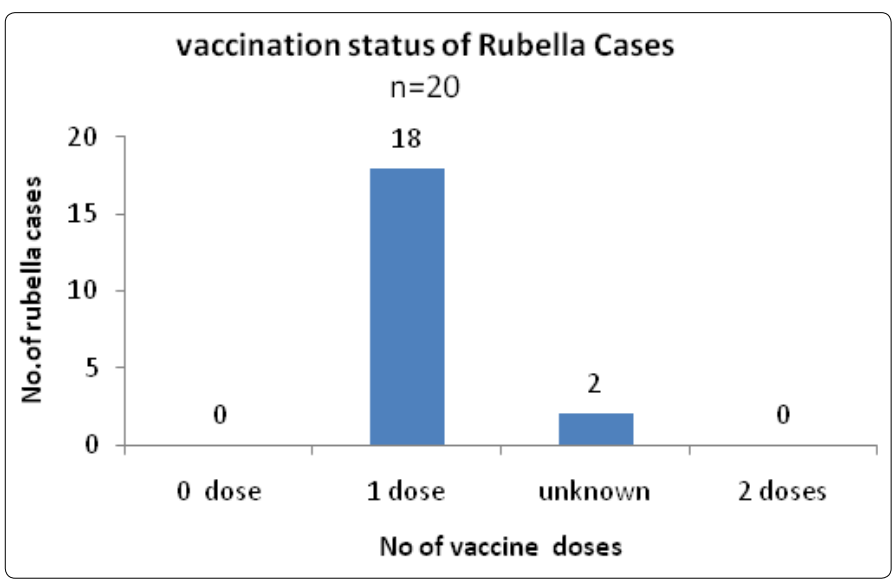

Figure 7. Vaccination status of rubella cases

Chi square test was used to determine the association between Recent MR vaccination and the occurrence of Rubella disease. The results indicated a strong association between the two (Chi 28.863, p 0.025).This indicates that if the cases got the MR vaccine during the campaign they would not have contracted the disease.

\section{Response to Rubella Outbreak}

Following reports of the suspected outbreak, The County Rapid Response team which is the subcommittee of the County Health Management Team (CHMT) was convened immediately to spearhead case definition, case search and case count, Determine the outbreak magnitude, orientate data by time, place and person, review cases, guide clinical management, coordinate proper specimen collection and referral to the laboratory, determine appropriate strategies, capacity building and control measures.

\section{Enhanced Surveillance}

Surveillance was intensified to ascertain the size and geographic extent of the outbreak. Health care workers in all Health facilities were alerted and encouraged to be vigilant in surveillance for rubella. Along with routine weekly reporting, Active case search was also carried out through regular visits to the health facilities and records review conducted to retrospectively find any cases that preceded the first reported case to help determine the time and circumstances of the beginning of the outbreak and better assess its full extent. Additional cases who met the operational case definition were referred to the health facilities for management, Line list was updated on daily basis and data analyzed by time (epi. curve) Place (mapping cases) and person (age sex and vaccination status). Daily reporting to the national level by $10 a . m$ of the following day and Monitor the effectiveness of outbreak response activity.

\section{Case Management}

An isolation ward was established to manage cases up to $4^{\text {th }}$ day from the date of onset of rash. In addition to treatment, 2 doses of vitamin A were given to the patients to prevent complications. Drugs, Vitamin A and other necessary supplies were provided. Clinical management guidelines/protocols were also supplied.

\section{Updating health staff skills}

Health workers were trained through on job training approach on case definition, case management procedures, reporting process and required data elements.

\section{Advocacy and social mobilization}

Social mobilization was done prior to the vaccination days. Advocacy meeting was held with the stakeholders. Letters were written to all the head teachers informing them of the outbreak, the need to immediately report cases to the local health Authorities, inform school children to pass the same message to their parents/guardians and the intended mop up vaccination targeting children 9 months to 14 years.

\section{MR Mop up campaign}

The team reviewed investigation results and interpreted data that had been analyzed. Based on the data the team concluded that Measles rubella mop up campaign be done in the entire Turi ward, Molo sub county targeting children 9 months to 14years.

Table 1. Results of MR Vaccination

\begin{tabular}{|c|c|c|c|c|c|}
\hline \multirow[b]{2}{*}{ Ward } & \multirow[b]{2}{*}{ Team / post } & \multirow{2}{*}{$\begin{array}{l}\text { Target Pop } \\
(9 \mathrm{~m}-14 \mathrm{Y}) \\
\text { as per Mi- } \\
\text { cro plan }\end{array}$} & \multicolumn{3}{|c|}{ Total Children Vaccinated } \\
\hline & & & $\begin{array}{l}9 \text { months } \\
59 \text { months }\end{array}$ & $\begin{array}{l}5 \text { years to } 14 \\
\text { years }\end{array}$ & $\begin{array}{l}\text { Total } \\
(9 \mathrm{Ms}-14 \mathrm{Yrs})\end{array}$ \\
\hline TURI & 1. St. Andrews & 1000 & 259 & 722 & 981 \\
\hline TURI & 2. Turi PCEA & 1130 & 265 & 907 & 1172 \\
\hline TURI & 3. Mitoni & 1000 & 301 & 1303 & 1604 \\
\hline TURI & 4. Nyeki & 1000 & 233 & 596 & 829 \\
\hline TURI & 5. Sagaitim & 1000 & 271 & 853 & 1124 \\
\hline TURI & 6. Ngwataniro & 1000 & 129 & 969 & 1098 \\
\hline TURI & 7. Chandera & 1000 & 249 & 702 & 951 \\
\hline TURI & 8. St Bredans & 1000 & 222 & 530 & 752 \\
\hline TURI & 9. Muchorwe & 1000 & 400 & 1076 & 1476 \\
\hline TURI & 10. Kiambireria & 1000 & 319 & 1273 & 1592 \\
\hline TURI & 11. Kayole & 1000 & 537 & 1044 & 1581 \\
\hline TURI & 12. Inooro & 1000 & 241 & 736 & 977 \\
\hline \multicolumn{2}{|c|}{ SUB COUNTY TOTALS } & 12130 & 3426 & 10711 & 14137 \\
\hline
\end{tabular}

\section{Conclusion}

Effective rubella surveillance system will lead to early case detection, early response and subsequently reduced rubella morbidity and mortality. Cases of parents resisting recommended vaccines for their children are on the increase 
especially with the increasing number of vaccination campaigns resulting in recurrence of vaccine preventable diseases threatening the gains made over the years especially on polio eradication, measles rubella control and elimination of neonatal tetanus. Motivating factors for Vaccine hesitant Parents (VHP) have not been thoroughly assessed. Establishing and understanding these factors will provide evidence-based approaches to address the challenge and will lead to increase in vaccine uptake for children of Vaccine Hesitant parents.

\section{Acknowledgements}

We would like to acknowledge the County Government of Nakuru for financial support, Kenya Medical Research Institute (KEMRI) measles laboratory for laboratory diagnosis, World Health organization (WHO) Kenya Western Region Office for Technical support, County Health Management Team Nakuru, Sub county Health Management Team Molo and all health workers for their unrelenting effort in responding to the said outbreak.

\section{Conflict of interest}

The authors declare that they have no Conflict of interest.

\section{References}

1. UNICEF. (2016, December 31). Retrieved June 1, 2017.

2. McLean $H$, Susan Redd $R$, Abernath $E$, Icenogle J. VPD Surveillance Manual. Rubella. 2012; 14-1.

3. WHO. (2017, March 1). WHO. Retrieved March 23, 2017.

4. Minstry of Public Health, Q. National Immunization Campaign for measles and rubella. Background Information about Measles,Mumps and Rubella(MMR) . Hamad, Hamad, Qatar, 2016.

5. Raju P. Rooting out Rubella:Making it mission Possible. Tamil Nadu, Goa, India, 2017.

6. Adedire E, Ajayi I, Fawole OI. Immunisation coverage and its determinants among children aged 12-23 months in Atakumosa-west district, Osun State Nigeria: a cross-sectional study. BMC Public health. 2016; 16(1): 905. doi: 10.1186/s12889-016-3531-x

7. Wang Z, Yan R, He H, Li Q, Chen E. Difficulties in Eliminating Measles and Controlling Rubella and Mumps: A Cross-Sectional Study of a First Measles and Rubella Vaccination and a Second Measles,Mumps, and Rubella Vaccination. PLOS ONE online. 2014; 9(2): e89361. doi: 10.1371/ journal.pone.0089361

8. Njeru I, Onyango D, Ajack Y, Kiptoo E. Rubella outbreak in a Rural Kenyan District, 2014: documenting the need for routine rubella immunization in Kenya. BMC Infect Dis. 2015; 15: 245. doi: 10.1186/ s12879-015-0989-6

9. $\mathrm{MOH}$. Guidelines for measles Surveillance and outbreak Response (Vol. Volume 2). Nairobi, Nairobi, Kenya: Ministry of Health, 2013.

10. Introducing rubella vaccine into national immunization programmes: a step by step guide. WHO. Geneva, Geneva, Switzerland: Expanded Programme on Immunization (EPI) of the Department of Immunization, Vaccines and Biologicals, 2015.

11. Maree DE. Kenya Rolls out massive Measles-Rubella and Tetanus campaign, 2015. 